\title{
Wider die Unsichtbarkeit:
}

\author{
Die tödliche Dimension rechter Gewalt im öffentlichen Raum
}

Heike Kleffner

In Koblenz und Hachenburg erinnern Initiativen auf unterschiedliche Weise an Todesopfer rechter und rassistischer Gewalt aus den 1990er Jahren. Diese Erinnerungsorte und ihre Entstehungsgeschichten sind ebenso wie die aktuellen Auseinandersetzungen um den Standort eines Mahnmals für die Opfer der rassistischen Bombenanschläge des NSU in Köln beispielhaft für das Sichtbarmachen der tödlichen Dimension von Rassismus und Rechtsextremismus im öffentlichen Raum.

Das Koblenzer Kulturforum ist ein imposanter Neubau auf dem zentralen Platz der rheinland-pfälzischen Stadt mit rund 112.00o Einwohner_innen. Eine unscheinbare Gedenkplatte unmittelbar im Eingangsbereich des strahlend weißen, den Platz weithin überragenden Gebäudes erinnert dort seit fünf Jahren an den tödlichen Amoklauf eines Neonazis vor einem Vierteljahrhundert. „Hier ermordete am 24.8.1992 ein rechtsradikaler Täter den Obdachlosen Frank Bönisch und verletzte mehrere Menschen. Zur Erinnerung und Mahnung“, lautet der Text auf dem schlichten Granitstein, der im Grau des Steinbodens auf dem Vorplatz am Kulturforum kaum auffällt. Die helle Schrift auf einer graubraun-gesprenkelten Steinplatte, an der täglich Dutzende Besucher_innen des Kulturforums achtlos vorbeigehen, ist trotz ihrer Unscheinbarkeit ein wichtiger Ort des Gedenkens an einen Amoklauf geworden, der schon zum Tatzeitpunkt kaum überregionales Aufsehen erregte. Und: Trotz der schlichten Gestaltung des Mahnmals und seiner Unscheinbarkeit zeigt die Entscheidung der Stadtverwaltung, die tödliche Dimension rechter Gewalt sichtbar zu machen, dass Zivilgesellschaft und Politik auf kommunaler Ebene oft weiter sind als Polizei und Justiz. Letztere verweigern dem ermordeten Obdachlosen Frank Bönisch seit Jahren die offizielle Anerkennung als Todesopfer rechter Gewalt. Obwohl die kleine Platte vor allem diejenigen zum Verweilen und Betrachten einlädt, die von ihrer Existenz wissen, haben die zwei Jahre andauernde öffentliche Debatte um ihre Verankerung sowie die jährlichen Gedenkveranstaltungen zum Jahrestag des neonazistischen Amoklaufs das Ziel der Initiator_innen erreicht: die knapp zwei Jahrzehnte in Vergessenheit geratene, tödliche Dimension 
neonazistischen Hasses gegen Wohnungslose und sozial Randständige sowohl im Stadtbild als auch im öffentlichen Diskurs zu verankern.

\section{Fürs, Vaterland': Blutbad im Herzen der Stadt}

An jenem warmen Augustabend 1992 war der Täter des Amoklaufs, Andy H., Spitzname „der deutsche Andy“, in Koblenz geblieben, während die meisten seiner Freunde von der „Deutschen Front Coblenz“ knapp 700 Kilometer gen Norden nach Rostock gefahren waren: Dort setzten in den späten Abendstunden des 24. August 1992 - nach zwei Tagen pogromartiger Angriffe gegen die Zentrale Aufnahmestelle für Asylsuchende im Stadtteil Rostock-Lichtenhagen - hunderte Naziskins und Neonaziaktivisten aus ganz Deutschland unter dem Beifall und den Anfeuerungsrufen von rund zweitausend Anwohner_innen und Schaulustigen ein Wohnheim ehemaliger vietnamesischer Vertragsarbeiter_innen in Brand. Nur durch einen glücklichen Zufall konnten sich die knapp einhundert Frauen, Kinder und Männer und ein ZDF-Kamerateam, die sich im so genannten „Sonnenblumenhaus“ aufhielten, vor den Flammen aufs Dach des Plattenbaus und dann in ein Nachbargebäude retten. Während sämtliche Fernsehsender die gewalttätige „Deutschland den Deutschen, Ausländer Raus“-Botschaft der Täter von Rostock quasi live in die Wohnzimmer der Republik übertrugen, führte der daheim gebliebene, damals 23-jährige Andy H. seinen eigenen Feldzug gegen alle, die nicht ins rechte Weltbild passen.

Der Dachdeckerlehrling entwendete eine Neun-Millimeter-Smith-\&Wesson-Pistole aus dem Waffenschrank seines Vaters und ging auf den Zentralplatz unweit des weltberühmten Deutschen Ecks. Dort saßen - wie an den vielen Sommerabenden Ende der 1980er Jahre - Punks, Junkies, Wohnungslose und sozial Randständige. Einige tranken Bier oder billigen Wein, ein paar spielten Gitarre. Der Zentralplatz war im Sommer ihr verlängertes Wohnzimmer - und anders als heute weder ein Ort des Konsums noch der ,Hochkultur . Andy H. - Hakenkreuz-Tätowierung auf dem Oberarm - stellte sich in Kampfschützenhaltung auf den Platz und brüllte: „Jetzt seid ihr dran“. Dann feuerte er zehn Schüsse, das gesamte Magazin, auf die völlig überraschten, wehrlosen Menschen ab. Acht Männer verletzte der Neonazi-Skinhead, einige von ihnen schwer. Der 35-jährige Obdachlose Frank Bönisch erlag noch vor Ort seinen Schussverletzungen.

Er sitze im Knast, weil er „dem Vaterland gedient“ habe, schrieb Andy H. nach seiner Festnahme aus der Haft in Briefen und Postkarten an „Kameraden“. Im Prozess vor dem Landgericht Koblenz im Juni 1993 jedoch gab sich der Lehrling, der davon träumte, Soldat oder Stuntman zu werden, reuig und unpolitisch. Als Auslöser für die Tat gab der 23-Jährige an, die Bank habe ihm am Vorabend einen Überziehungskredit von 100 Mark verweigert. Da habe er mit allem Schluss machen wollen. Er habe sich als Auserwählter gefühlt, der dazu bestimmt sei, Menschen zu töten. Ein psychiatrischer Gutachter bescheinigte Andy H. laut Urteil eine ,schwere Persönlichkeitsstörung, Minderwertigkeitsgefühle, Angst und Hass “ und hielt ihn für vermindert schuldfähig. Das Landgericht Koblenz verurteilte Andy H. schließlich wegen Mordes und siebenfachen Mordversuchs zu einer Freiheitsstrafe von 15 Jahren und ordnete seine Einweisung in eine psychiatrische Landesanstalt an. Ein 
politisches Motiv für die Schüsse erkannten die Richter genauso wenig wie die Staatsanwaltschaft. Der Journalist Michael Grabenströer, der für die Frankfurter Rundschau über den Prozess berichtete, schrieb daraufhin: „In der Garnisons- und Beamtenstadt Koblenz wurde die Tat nur zu gerne als Amoklauf ohne politischen Hintergrund gesehen." (Grabenströer 1992)

\section{Wohnungslose und sozial Randständige als Opfer rechter Gewalt sichtbar machen}

Dass auch die Landes- und die Bundesregierung den Obdachlosen Frank Bönisch nicht als Opfer politisch rechts motivierter Gewalt anerkennen, hatte der langjährige Kulturdezernent der Stadt Koblenz, Detlef Knopp, eher am Rande zur Kenntnis genommen. Nachdem Knopp, vor seinem Amtsantritt als Kulturdezernent im Schuldienst als Sozialkunde- und Politiklehrer beschäftigt, das Gerichtsurteil und die Medienberichte über das Verfahren gelesen hatte, sprach er in Interviews vor der Gedenksteinlegung im Sommer 2012 gegenüber Journalist_innen von einer eindeutigen, „objektiven Faktenlage“: Der Mord an Frank Bönisch sei aus „Gesinnungsgründen“ geschehen, die Opferauswahl füge sich nahtlos ins klassische rechtsradikale Weltbild. Für ihn sei es daher selbstverständlich gewesen, den Mahnmals-Antrag eines zivilgesellschaftlichen Bündnisses aus einer lokalen Obdachloseninitiative, dem DGB, der Katholischen Hochschulgemeinde und jungen AntifaAktivist_innen zu unterstützen. Die Gruppe hatte im Vorfeld intensiv mit Zeitzeug_innen, Freund_innen des Getöteten und verletzten Überlebenden des Attentats gesprochen. „Obwohl das Attentat und der Mord an Frank Bönisch fast zwei Jahrzehnte zurücklag, als wir mit unseren Recherchen begannen, sind die Überlebenden immer noch mit den Folgen konfrontiert“, sagt Sebastian Hebeisen vom DGB in Koblenz, wenn man mit ihm heute über die Entstehungsgeschichte des Mahnmals spricht. Hebeisen ist einer der Sprecher der Initiative und betont: „Niemand hatte jemals ernsthaft mit den Überlebenden und Verletzten des Attentats über ihre Ängste und ihre Trauer geredet, sie waren eigentlich komplett unsichtbar.“[1]

Einige Mitglieder der späteren Initiative in Koblenz hatten zuvor an einer Gedenkveranstaltung der 50 Kilometer von Koblenz entfernten Kleinstadt Hachenburg im Westerwald für den im Dezember 1990 von Neonazis getöteten 17-jährigen kurdischen Flüchtling Nihad Yusufoğlu teilgenommen (Roggenkamp 1991). „Dabei wurde eine Liste von rund 150 Todesopfern rechter Gewalt an eine Hauswand projiziert“, erinnert sich Hebeisen. Dort sei ihnen dann der Mord an Frank Bönisch aufgefallen, der ja quasi „direkt vor der eigenen Haustür“" geschehen sei. Intensive Recherchen und viele Archivbesuche, ein Open-Air-Gedenkkonzert, eine Demonstration und schließlich im Juli 2013 die Einweihung des Gedenksteins folgten.

„Wir wollen mit der Tafel auch daran erinnern, dass rechte und rassistische Gewalt weiterhin ein großes Problem ist - und eine ansonsten oftmals unsichtbare Opfergruppe, Obdachlose und sozial Randständige, sichtbar machen“,

sagt Hebeisen. Hass auf sozial Randständige und eine tief sitzende Verachtung für Wohnungslose seien ein zentrales Motiv rechter Gewalttäter, das noch 
dazu tief in der Mitte der Gesellschaft wurzele. Unter den 169 Todesopfern rechter Gewalt seit 1990, die von Tagesspiegel und ZEIT Online recherchiert wurden, sind neben Frank Bönisch weitere 38 Obdachlose und Menschen, die von den Tätern als am Rand der Gesellschaft stehend wahrgenommen wurden (Jansen et al. 2018a; b). Die Mehrheit von ihnen ist - wie Frank Bönisch aus Koblenz - nicht offiziell von der Bundesregierung als Todesopfer rechter Gewalt anerkannt. Sebastian Hebeisen sagt, in den Gesprächen mit den Verletzten des Attentats sei diese mangelnde staatliche Anerkennung für „den tödlichen, in direkter Tradition des historischen Nationalsozialismus stehenden rechten Hass auf vermeintlich lebensunwertes Leben" immer wieder als „Schlag ins Gesicht“ bezeichnet worden. Schließlich spiegele sich darin eine Erfahrung von Missachtung, die viele Punks, vermeintliche ,Gammler', ,Bettler' und Bezieher_innen staatlicher Transferleistungen mit Vertreter_ innen von Behörden und staatlichen Institutionen ohnehin machen würden. Umso wichtiger sei es, dass die Lokalpolitik sich mit der Entscheidung für die Gedenkplatte deutlich von der „Politik der Unsichtbarkeit der Opfer“ abgegrenzt habe.

\section{Jährliche Gedenkveranstaltungen als diskursive Orte}

Die jährlichen Erinnerungsveranstaltungen an das Attentat und an Frank Bönisch, die die Initiative seit inzwischen sechs Jahren am Tatort auf dem Platz vor dem Kulturforum organisiert, seien inzwischen „wichtige Kristallisationspunkte für einen kommunalen Austausch über aktuelle Erscheinungsformen rechter Gewalt und Organisierung geworden“, betont Hebeisen. Auch in der Kleinstadt Hachenburg erfüllen die jährlichen Gedenkveranstaltungen für den am 28. Dezember 1990 von einem in der „Taunusfront“ organisierten Neonazi erstochenen Nihad Yusufoğlu eine wichtige Funktion. Seit einigen Jahren nimmt die Familie des getöteten kurdischen Jugendlichen, die nach dem Verlust ihres Sohnes nach Norddeutschland gezogen ist, die Einladungen der Stadt und der örtlichen Kirchengemeinde an. Sie versammelt sich mit Einwohner_innen aus Hachenburg vor dem Fachwerkhaus, vor dem der 17-Jährige starb. Direkt gegenüber hängt an der Wand des städtischen Parkhauses eine weithin sichtbare schwarze Gedenkplatte. Deren Botschaft ist unübersehbar: „Zum Gedenken an Nihad Yusufoğlu, am 28.12.1992 von einem Neo-Nazi erstochen. ,Erinnern heißt widerstehen““.

Auch Nihad Yusufoğlu gehört - wie Frank Bönisch - zu der Mehrheit der mindestens 169 Todesopfer rechter, rassistischer und antisemitischer Gewalt, die von der Bundesregierung nicht offiziell anerkannt werden. Umso wichtiger sei der Familie das sichtbare lokale Gedenken, sagt Sebastian Hebeisen:

„Vielen Überlebenden rechter Gewalt und Hinterbliebenen ist die Erinnerung an ihre traumatischen Erfahrungen wieder sehr nah, seitdem bundesweit die Zahl der rassistischen Angriffe auf Flüchtlinge wieder so massiv zugenommen hat wie zuletzt in den 1990er Jahren.“

Zumal es noch weitere Parallelen gebe: Noch immer werde rechte, rassistische und antisemitische Gewalt ausschließlich in Ostdeutschland verortet. 
Und: Vielerorts würden sich die Täter_innen durch offen rechte Social Media Plattformen, Filterblasen und Hetzkampagnen von AfD \& Co. legitimiert fühlen.

Im zunehmend polarisierten gesellschaftlichen Klima sei es notwendig, dass Orte, Räume und Anlässe geschaffen würden, um den Betroffenen rechter und rassistischer Angriffe zuzuhören, sagt auch Judith Porath vom Verein Opferperspektive. Sie berät seit knapp zwei Jahrzehnten Betroffene rechter Gewalt und deren Angehörige in Brandenburg. „Die Antwort auf die Frage, welche Sorgen welcher Bürger_innen ernst genommen und das Stadtgespräch beherrschen“, dürfe nicht einer lautstarken, gut organisierten Minderheit von „Flüchtlingshassern“ überlassen werden. Bürgerbündnisse und Initiativen, die sich für ein aktives Gedenken an Todesopfer rechter Gewalt einsetzen, rührten daran, dass viele Gemeinden und die meisten ihrer Bewohner_innen die Taten am liebsten bis heute verdrängen möchten. „Sie werfen damit natürlich gleichzeitig die Frage auf, wie das Zusammenleben in der jeweiligen Kommune heute aussieht“, sagt Porath.[2] Die häufig bis heute fortdauernde Dominanz extrem rechter Strukturen und die Ausgrenzung von Randgruppen würden dann zum Thema:

„Die Orte der tödlichen Dimension rechter, rassistischer und antisemitischer Gewalt deutlich zu kennzeichnen - sei es durch Stolpersteine in Erinnerung an die durch die nationalsozialistische Shoa ermordeten Juden und Jüdinnen oder durch Gedenksteine und Orte etwa für die Opfer des ,Nationalsozialistischen Untergrunds' (NSU) - ist notwendig, um die Konsequenzen von gesellschaftlichen Konstruktionen in ,Die Anderen und Wir im lokalen und stadtpolitischen Alltag für alle sichtbar zu machen und zu bearbeiten.“

betont Judith Porath. Sie verweist auf den aktuellen Konflikt in Köln. Das von den Überlebenden der rassistischen Bombenanschläge in Köln geforderte Mahnmal müsse zum 15. Jahrestag des verheerenden NSU-Nagelbombenanschlags in der Keupstraße am 10. Juni 2019 müsse endlich an dem von den Betroffenen gewünschten Standort - für alle gut sichtbar - errichtet werden.

\section{Endnoten}

[1] Interview der Autorin mit Sebastian Hebeisen am 10. September 2018.

[2] Interview der Autorin mit Judith Porath am 8. September 2018.

\section{Autor_innen}

Heike Kleffner ist Journalistin und Geschäftsführerin des Verbands der Beratungsstellen für Betroffene rechter, rassistischer und antisemitischer Gewalt (VBRG e.V.). Gemeinsam mit Frank Jansen vom Tagesspiegel betreut sie seit September 2000 das Langzeitrechercheprojekt „Todesopfer rechter Gewalt seit 1990“ für Tagesspiegel und Zeit/Zeit Online.

h.kleffner@verband-brg.de 


\section{Literatur}

Grabenströer, Michael (1992): Eine Kleinstadt im Westerwald und ein Messer im Stadtbrunnen. In: Frankfurter Rundschau, 26.2.1992.

Jansen, Frank / Kleffner, Heike / Staud, Toralf / Radke, Johannes (2018a): Todesopfer rechter Gewalt: 169 Schicksale. In: ZEIT Online, 28.9.2018. www.zeit.de/gesellschaft/ zeitgeschehen/2018-09/todesopfer-rechte-gewalt-karte-portraet (letzter Zugriff am 12.2.2019).

Jansen, Frank / Kleffner, Heike / Staud, Toralf / Radke, Johannes (2018b): Erstochen, erschlagen, verbrannt. In: ZEIT Online, 28.9.2018. www.zeit.de/gesellschaft/zeitgeschehen/2018-09/rechtsxtremismus-todesopfer-gewalt-verdacht (letzter Zugriff am 12.2.2019).

Roggenkamp, Viola (1991): Sie johlten und grölten, als Nihat kam. In: DIE ZEIT 6/1991. www. zeit.de/1991/o6/sie-johlten-und-groelten-als-nihat-kam (letzter Zugriff am 12.2.2019). 